www.conferenceie.ase.ro

\title{
MOBILE DATA VULNERABILITIES
}

\author{
Alin ZAMFIROIU \\ Bucharest University of Economic Studies, Romania \\ alin.zamfiroiu@csie.ase.ro \\ Paul POCATILU \\ Bucharest University of Economic Studies, Romania \\ ppaul@ase.ro \\ Sergiu CAPISIZU \\ Bucharest Bar Association \\ sergiu.capisizu@yahoo.com
}

\begin{abstract}
Mobile devices are very popular today and also the mobile applications market. The mobile operating market is shared among Android and iOS. Mobile applications are built natively for these operating systems or are Web-based. No matter the platform, there are user data managed by the mobile applications and transferred on external servers. These data are exposed to attacks and the developers should be always concerned about the security issues related to their systems. In this paper we analyze the vulnerabilities related to mobile applications and we propose several countermeasures, in order to eliminate or to mitigate the security risks.
\end{abstract}

Keywords: Common Vulnerabilities and Exposures (CVEs), Mobile, Data, Security, Vulnerability.

JEL classification: L86, C88

DOI: $10.12948 / \mathrm{ie} 2019.06 .10$

\section{Introduction}

Almost all mobile applications use cloud storage because it saves data on your mobile device, reduce cost, and provide reliability and support disaster recovery [1], [2]. These data have to be protected against the unauthorized access. Security for mobile devices and for mobile applications is provided in different areas of interest such as:

- authentication, with verification of virtual identity, by requesting personal authentication information and verifying them with previously provided information at the time of registration. According to [3] authentication is the process by which the correctness and truthfulness of the information relating to the identity or origin of an entity is established or confirmed.

- communication or text messaging is one of the core business of mobile devices as well as transactions. This is a very important segment where security is vital to protect mobile device users. In [4] the SMSEncrypt application for people who want secure communication via the SMS service provided by the mobile phone company is presented. The SMSEncrypt software is composed of two modules: a module for sending encrypted messages with the specified key and the second module for receiving messages and decryption with the secret key the message receiver needs to know.

- the information is stored in the cloud as a backup for mobile device destruction; In the device's internal memory, the user stores photos taken with this device, text messages used to communicate with others, emails saved in the phone's memory, other people's contacts, 
www.conferenceie.ase.ro

or contact list; all this information in case of damage to or destruction of the mobile device is lost and its recovery is impossible or very difficult; it is recommended that you keep a backup in the cloud [5];

- encrypting the information stored locally for the case when the device is lost or stolen by others, so personal information is at their disposal; so the information that the previous recommendation is saved in a cloud backup is stored on the device but protected by a password, access to which is only allowed by specifying the encryption key.

In all these areas, the security should be assured for the used data through the mobile application, because the data is used in all areas.

\section{Related work}

In their paper, Altuwaijri and Ghouzali [6] presented a threat model for storing mobile device data of Android based devices, Figure 1. shows this threat model. Altuwaijri and Ghouzali threat model classify threats into Physical and Software threats.

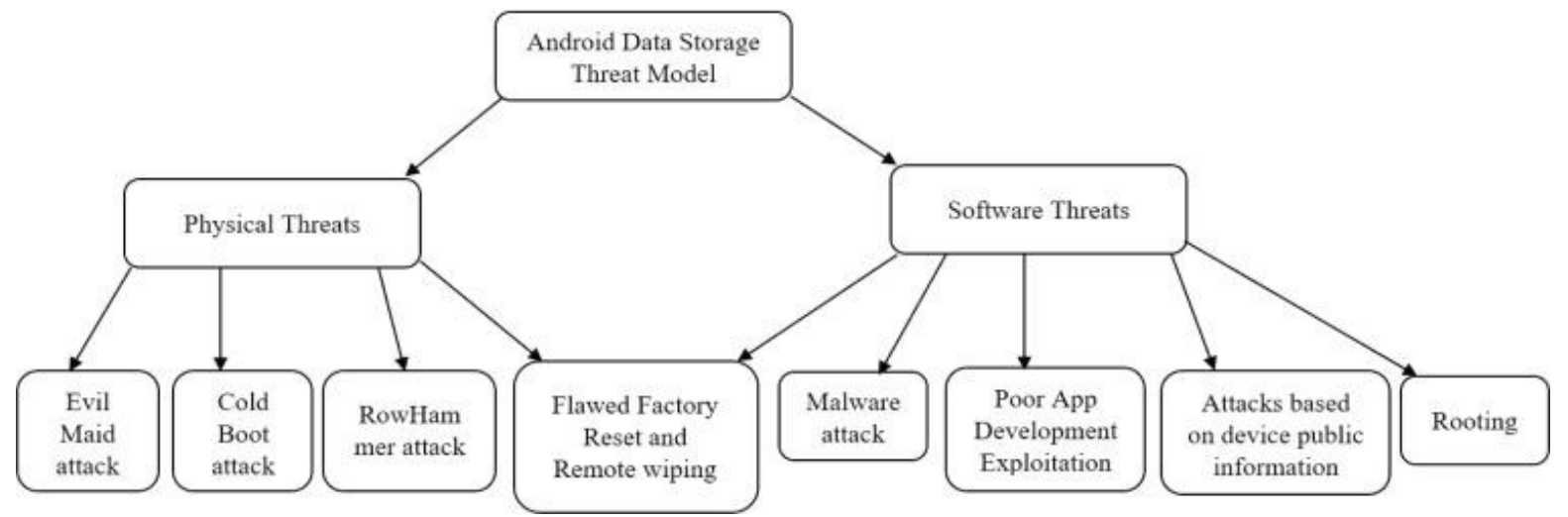

Figure 1. Threat model for storing data in Android [6]

Physical threats are possible when mobile devices get into the hands of unauthorized people through loss or theft. These are three types [6]:

- Cold boot attack - assumes that ram memory is obtained and connected to another device to obtain data from that memory;

- Evil maid attack - assumes the unencrypted memory area; even if a password is used to encrypt data on your mobile device, the Android partitioning system can be modified with keylogging;

- RowHammer attack - assumes the use of management vulnerability at the lower levels of the operating system; is the most complex vulnerability of physical.

Software threats include malware attacks or poor mobile application development [7]:

- Malware attack - involves installing applications that contain malware, which then develops into the infected mobile device;

- Poor app development - in this category, developers' level of knowledge of security concepts and how they develop mobile applications; if developers do not implement community-approved security standards, the developed application will be very vulnerable in terms of security.

- Attacks based on device public information - involves knowledge of mobile devices and applications running on these devices, and attackers use these vulnerabilities to perform attacks;

- Routing the device to remove limitations imposed by the developer leads inevitably to the emergence of new vulnerabilities that can be exploited by attackers. 
OWASP, the nonprofit group that helps companies develop and service security applications issued in 2016 a document with the most used vulnerabilities for mobile applications. These are shown in Figure 2.

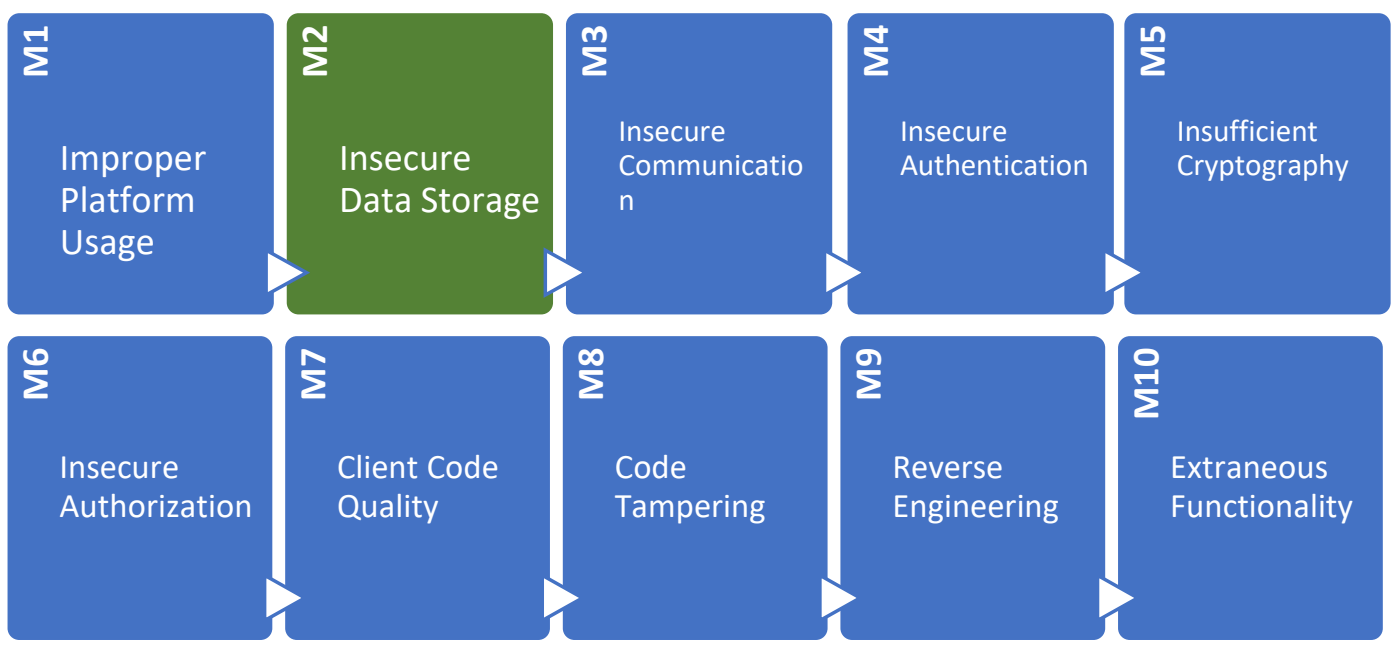

Figure 2. OWASP Mobile Top 10 Risks [8]

For each vulnerability, the ways to check if mobile applications are vulnerable and how to prevent these vulnerabilities.

\section{Common Vulnerabilities and Exposures of Mobile Data}

Common Vulnerabilities and Exposures [8] provide a reference method for publicly known vulnerabilities and exposures. Within the CVE vulnerability base are stored all the important IT vulnerabilities discovered so far. Shortly after a new vulnerability is discovered, it is documented and receives a new unique identifier in the CVE list. Currently, the CVE vulnerability base is a reference source for cyber security specialists.

In order to select only CVEs relevant to mobile app data, in this paper, we started with a list $f$ mobile-specific keywords and used them to search for the common vulnerabilities. As mobile devices as well as mobile applications have a rapid increase but also a high degree of change, CVEs are selected in the last 2 years: 2017-2018.

Table 1 lists CVEs relevant to mobile application data, grouped by key terms used for search.

Table 1. CVEs specific for mobile data

\begin{tabular}{|c|c|c|c|}
\hline Nr. & Keyword & $\begin{array}{c}\text { Number of CVEs found } \\
(2017-2019)\end{array}$ & $\begin{array}{c}\text { Total number of } \\
\text { CVEs found }\end{array}$ \\
\hline 1 & mobile applications & 16 & 27 \\
\hline 2 & mobile data & 39 & 89 \\
\hline 3 & sensitive data & 363 & 1088 \\
\hline 4 & Android data & 196 & 409 \\
\hline 5 & phone data & 10 & 24 \\
\hline 6 & iOS data & 40 & 127 \\
\hline 7 & device data & 117 & 242 \\
\hline
\end{tabular}

For the 7 terms used, a total of 2006 CVEs were identified, out of which 781 were documented in the last years, 2017-2019.

For the term "sensitive data", most CVEs have been identified, as is also shown in Figure 3. 


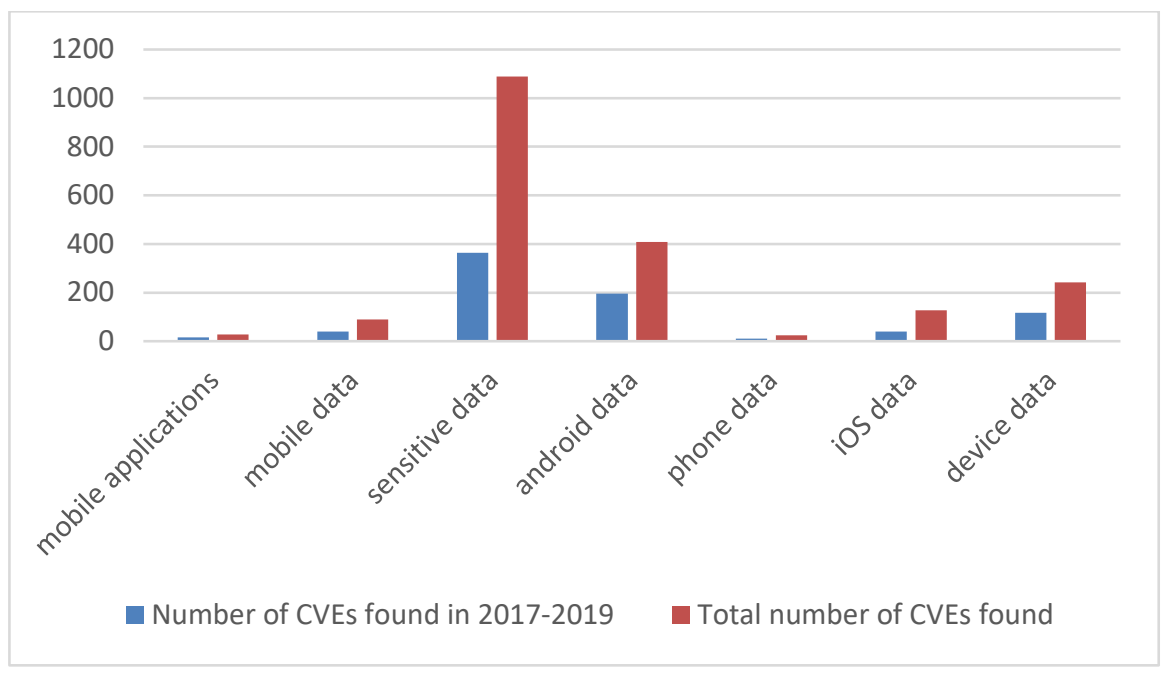

Figure 3. Identified CVEs for Mobile Data

Of the 781 CVEs identified between 2017 and 2019, 107 fall into two or more categories. Table 2 lists CVEs that are found in 3 or 4 categories. To identify the common vulnerabilities for all categories we used a word counter as in [10].

Table 2. Common Vulnerabilities per categories

\begin{tabular}{|c|c|c|c|}
\hline No. & CVE & $\begin{array}{l}\text { Number of } \\
\text { categories }\end{array}$ & Categories \\
\hline 1 & cve-2018-4844 & 4 & mobile data, Android data, iOS data, device data \\
\hline 2 & cve-2018-6599 & 4 & sensitive data, Android data, phone data, device data \\
\hline 3 & cve-2018-4847 & 4 & mobile data, sensitive data, iOS data, device data \\
\hline 4 & cve-2018-14995 & 4 & sensitive data, Android data, phone data, device data \\
\hline 5 & cve-2017-18125 & 3 & mobile applications, mobile data, Android data \\
\hline 6 & cve-2017-10188 & 3 & mobile applications, mobile data, Android data \\
\hline 7 & cve-2017-12228 & 3 & sensitive data, iOS data, device data \\
\hline 8 & cve-2018-14984 & 3 & Android data, phone data, device data \\
\hline 9 & cve-2017-10132 & 3 & mobile applications, mobile data, iOS data \\
\hline 10 & cve-2018-14985 & 3 & mobile data, Android data, device data \\
\hline 11 & cve-2018-15005 & 3 & mobile data, Android data, device data \\
\hline 12 & cve-2018-14987 & 3 & mobile data, Android data, device data \\
\hline 13 & cve-2018-0461 & 3 & sensitive data, phone data, device data \\
\hline 14 & cve-2017-17225 & 3 & mobile data, phone data, device data \\
\hline 15 & cve-2018-4168 & 3 & sensitive data, iOS data, device data \\
\hline
\end{tabular}

Thus, out of the total of 781 CVEs, the unique ones are 655 . Figure 4 shows the relationships between the category vulnerabilities: \{device data, mobile data, iOS data, mobile applications . Only these five categories were chosen because the other two \{Android data, sensitive data\} contain many elements and the visibility would not have been of any quality. 


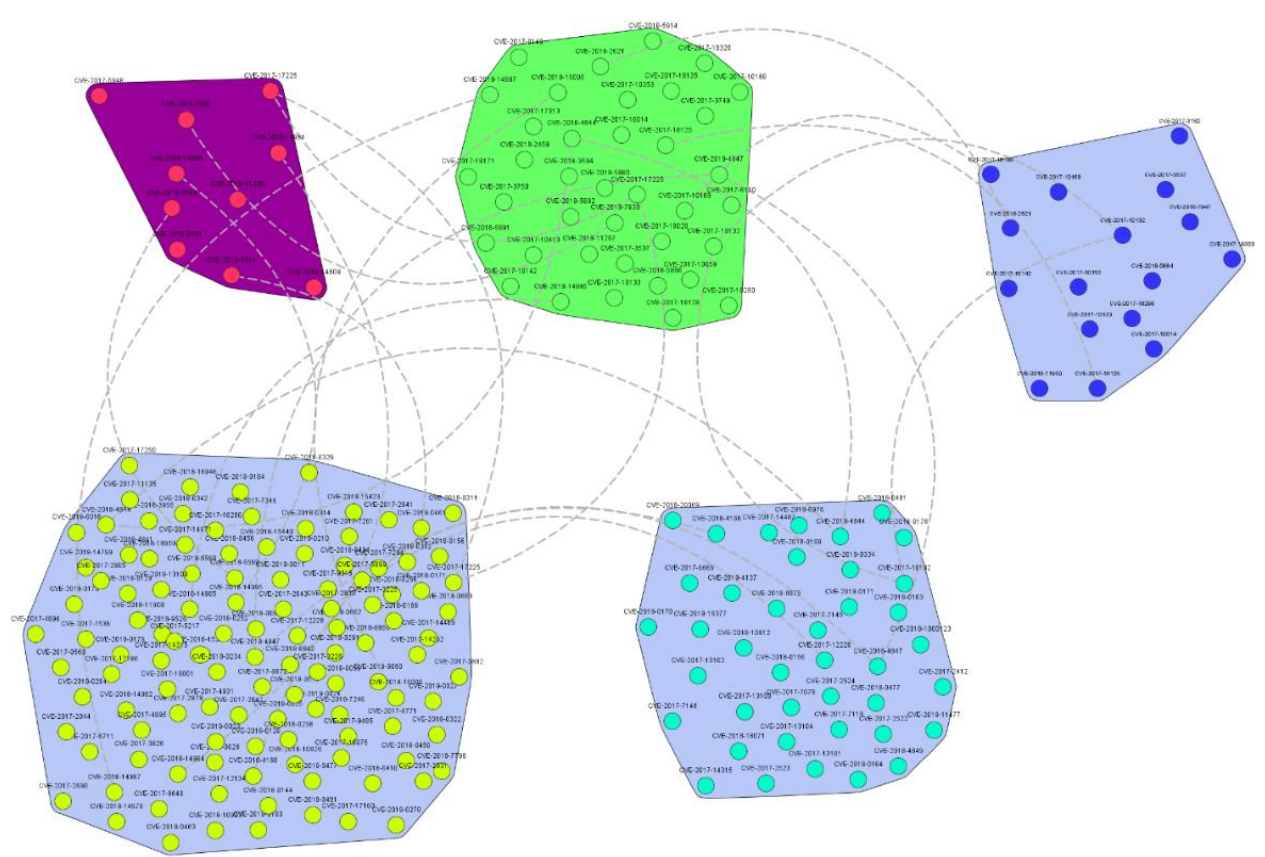

Figure 4. The links between the vulnerabilities of the categories

The list of CVEs is updated daily, so there are certainly more vulnerabilities for data used in mobile applications.

\section{Countermeasures}

In [6] there are some solutions for mobile devices running Android OS. These are presented in Table 3.

Table 3. Hardware solutions for data security on mobile devices [6]

\begin{tabular}{|c|c|l|}
\hline No. & Solution & \multicolumn{1}{c|}{ Implemented by: } \\
\hline 1 & CleanOS & Xia Y., Liu Y., Ma m., Guan H., Zang B., Chen H. \\
\hline 2 & TinMan & $\begin{array}{l}\text { Yubin Xia, Yutao Liu, Cheng Tan, Mingyang Ma, Haibing Guan, Binyu } \\
\text { Zang, Haibo Chen }\end{array}$ \\
\hline 3 & Sentry & $\begin{array}{l}\text { Colp p., Zhang J., Gleeson J., Suneja S., Lara E., Raj H., Saroiu S., } \\
\text { Wolman A. }\end{array}$ \\
\hline 4 & Armored & Muller T. Spreitzenbarth M. \\
\hline 5 & Deadbold & Skillen A., Barrera D., Oorschot P. \\
\hline 6 & Droidvault & Li X., Hu H., Bai G., Jia Y., Liang Z., Saxena P. \\
\hline 7 & RPMB & Reddy A.K., Paramasivam P., Vemula P.B. \\
\hline 8 & CATT & Brasser F., Davi L., Gens D., Liebchen C., Sadeghi A. \\
\hline 9 & ARMOR & Ghasempour M., Lujan M., Garside J. \\
\hline
\end{tabular}

From a software point of view, there are two options: FDE (Full Disk Encryption) and KeyChain available from Android 4.4 (API 19) and Android 4.0 (API 14), respectively.

In literature, several countermeasures have been identified for vulnerabilities in the wild, as well as for vulnerabilities found for data used in mobile applications. For example, in [11] there are presented compromise measures for mobile operating systems such as:

- controlled and conditional access to the applications;

- restrictions and user blocking in applications to identify different behaviors;

- monitoring, reporting, and reporting for all user actions in mobile applications;

- achieving accurate procedure for incident response mode; 
- updating operating systems on mobile devices;

- encrypt the mobile data;

- using pins, passwords, and access codes.

Vulnerabilities will always exist, and new ones will be discovered. The goal of mobile application data security is to identify these vulnerabilities to eliminate vulnerabilities discovered and to be prepared for new vulnerabilities.

\section{Conclusions}

In this paper, we introduced a new approach for identifying the vulnerabilities and exposures that are the most common and represent the highest threat on mobile data. In order to identify those vulnerabilities we started from the list of the generic Common Vulnerabilities and Exposures (CVEs) that are normally used by security experts, and used a search based approach in which we started by a list of common words related to mobile data and based on the retrieved documents, we identified the common vulnerabilities in these documents. After that, we studied the relationship between the extracted vulnerabilities and the different search categories. Accordingly, we proposed a number of countermeasures that the security experts need to consider in order to keep the mobile data secure.

\section{Acknowledgment}

This paper presents results obtained within the PN-III-P1-1.2-PCCDI-2017-0272 ATLAS project ("Hub inovativ pentru tehnologii avansate de securitate cibernetică/Innovative Hub for Advanced Cyber Security Technologies"), financed by UEFISCDI through the PN III "Dezvoltarea sistemului national de cercetare-dezvoltare", PN-III-P1-1.2-PCCDI-2017-1 program.

\section{References}

[1] T. H. Noor, S. Zeadally, A. Alfazi, Q. Z. Sheng, "Mobile cloud computing: Challenges and future research directions" Journal of Network and Computer Applications 115 (2018), pp. 70-85.

[2] A. S. M. E. Yuksel, A. Sertbas, A. H. Zaim, "Implementation of a web-based service for mobile application risk assessment," Turk J Elec Eng \& Comp Sci (2017) 25: 976 - 994 TUBITAK, doi:10.3906/elk-1503-127.

[3] M. Marian, Ghid de Securitate Informatică, Craiova: Universitară Publishing House, 2009.

[4] C. Boja, P. Pocatilu and A. Zamfiroiu, "Data Security in M-Learning messaging services," International Journal of Computer Communications, vol. 5, pp. 119-126, 2011.

[5] B. Iancu, T.M. Georgescu, "Saving Large Semantic Data in Cloud: A Survey of the Main DBaaS Solutions", Informatica Economică vol. 22, no. 1/2018

[6] H. Altuwaijri and S. Ghouzali, "Android data storage security: A review," Journal of King Saud University - Computer and Information Sciences, In Press, Corrected Proof, Available online: 19 July 2018, DOI: 10.1016/j.jksuci.2018.07.004.

[7] M. Sujithra, G. Padmavathi and S. Narayanan, "Mobile Device Data Security: A Cryptographic Approach by Outsourcing Mobile data to Cloud," Procedia Computer Science 47 (2015), pp. $480-485$.

[8] OWASP 2016, https://www.owasp.org/index.php/OWASP_Mobile_Security_Project

[9] CVE - Common Vulnerabilities and Exposures, 2019, https://cve.mitre.org/

[10] Wordcounter, https://wordcounter.com/, [February 9, 2019]

[11] M. Raggo, Mobile Data Loss: Threats and Countermeasures, Syngress, 16th December 2015, eBook ISBN: 9780128029015, pp. 55, [February 9, 2019]. 